\title{
Systemic immune-inflammation index predicts prognosis of sequential therapy with sorafenib and regorafenib in hepatocellular carcinoma
}

\section{Young Mi Hong}

Pusan National University Yangsan Hospital https://orcid.org/0000-0001-5427-7505

\section{Ki Tae Yoon}

Pusan National University Yangsan Hospital

Mong Cho ( $\sim$ mcho@pusan.ac.kr)

Pusan National University Yangsan Hospital

\section{Research article}

Keywords: regorafenib, hepatocarcinoma carcinoma, inflammation, prognosis, systemic immuneinflammtaion index, alpha-fetoprotein

Posted Date: November 9th, 2020

DOl: https://doi.org/10.21203/rs.3.rs-46929/v2

License: (c) (i) This work is licensed under a Creative Commons Attribution 4.0 International License. Read Full License 


\section{Abstract}

Background: Regorafenib has shown promising results as a second-line therapy for patients with hepatocellular carcinoma (HCC) who progressed on sorafenib. Although several data regarding the efficacy of sequential therapy with sorafenib and that of regorafenib in real-life, specific inflammation markers for predicting the prognosis have not been studied. This study aimed to investigate prognostic value of systemic inflammatory markers in patients with HCC who received sorafenib-regorafenib sequential therapy.

Methods: We retrospectively analyzed medical data of patients who received regorafenib for the treatment of HCC after sorafenib failure. Progression free survival (PFS) and overall survival (OS) were assessed using the Kaplan-Meier survival curves. Univariate and multivariate analyses were performed to analyze the factors associated with survival.

Results: A total of 58 patients who received at least one dose of regroafenib and fulfilled the eligibility criteria, good performance status (Eastern Cooperative Oncology Group [ECOG] 0-1) and preserved liver function (Child-Pugh-A), were included in the analysis. The median PFS was 3 months (95\% confidence interval $[\mathrm{Cl}]=0.981-5.019)$ and the median OS was 8 months $(95 \% \mathrm{Cl}=5.761-10.239)$. Elevated systemic immune-inflammation index (SII $\geq 340$ ) was independently associated with poor OS. In multivariate analysis, the SII (hazard ratio $[\mathrm{HR}]=2.211,95 \% \mathrm{Cl}=1.089-4.489, \mathrm{P}=0.028$ ) and alpha-fetoprotein (AFP) $(\mathrm{HR}=2.750,95 \% \mathrm{Cl}=1.259-6.010, \mathrm{P}=0.011)$ were independent predictors of OS.

Conclusion: Elevated SII is associated with poor OS in patients with HCC who received sequential therapy with sorafenib and regorafenib. In addition, when selecting a treatment strategy, the SIl can be used in combination with the AFP level as a promising prognostic tool for HCC.

\section{Background}

Hepatocellular carcinoma (HCC), one of the most common cancers, is the third leading cause of cancerrelated mortality worldwide [1]. Despite the advancements in diagnosis and surveillance programs for $\mathrm{HCC}$, the majority of patients are diagnosed in an advanced stage, thus being unsuitable to potentially curative treatments such as surgery, liver transplantation, or ablative therapy. Systemic treatment is recommended for patients with advanced HCC at diagnosis or with unresectable HCC who cannot benefit from locoregional therapy. Sorafenib, a multikinase inhibitor, is the first approved systemic chemotherapy for advanced HCC [2]. Since the approval of sorafenib in 2008, no second-line agents following sorafenib treatment were available for a fairly long time. Recently, regorafenib [3], cabozantinib [4], nivolumab [5], pembrolizumab [6], and ramucirumab $[7,8]$ have been approved as a second-line systemic chemotherapies for advanced HCC.

Regorafenib is an oral multikinase inhibitor that targets vascular endothelial growth factor receptors (VEGFRs) 1-3, KIT, RET, RAF-1, BRAF, platelet-derived growth factor receptor (PDGFR), fibroblast growth factor receptor (FGFR), and colony-stimulating factor 1 receptor (CSF1R) [9-11]. It has been adopted as an 
initial second-line agent and sorafenib-regorafenib sequential treatment in patients with advanced HCC. Although several studies have reported outcomes of sorafenib-regorafenib sequential treatment, few studies have been evaluated the prediction of survival.

Inflammation plays an essential role in tumor development and tumor metastasis, and immune surveillance plays a crucial role in cancer elimination $[12,13]$. Recently, many studies have reported that combined scores using peripheral inflammatory cells (neutrophils, lymphocytes, and platelets) are associated with survival in various tumors [14-17]. To the best of our knowledge, however, no studies regarding systemic inflammatory markers in predicting HCC patients received sequential therapy with sorafenib and regorafenib. Therefore, we aimed to determine the prognostic value of systemic inflammatory markers in patients with advanced HCC.

\section{Methods}

\section{Patient eligibilityand regorafenib treatment}

We retrospectively reviewed the data of patients who received regorafenib between July 2017 and April 2020. The diagnosis of HCC was based on American Association for the Study of Liver Diseases criteria. Patients who failed sorafenib treatment, which was confirmed based on radiological progression during sorafenib therapy, were enrolled. Inclusion criteria were in accordance with hase 3 trial of regorafenib (RESORCE trial) and were as follows: tolerability to sorafenib ( $\geq 400 \mathrm{mg}$ daily for at least 20 of the 28 days) and preserved liver function (Child Pugh A). Regorafenib was prescribed in a 4-week cycles, with a starting dose of $160 \mathrm{mg}$ once per day for 3 weeks and 1 week of no treatment. Dose reductions and interruption of regorafenib were performed based on occurrence of adverse events [3]. Regorafenib treatment was continued until patients experienced intolerable adverse events or until confirmation of radiologic progressive disease (PD). This retrospective study was reviewed and approved by the Ethical Committee of our center.

\section{Clinical variables}

Clinical data such as baseline demographics, Eastern Cooperative Oncology Group performance status, complete blood count, alpha-fetoprotein (AFP), protein induced by vitamin $\mathrm{K}$ absence or antagonist II (PIVKA-II), Child-Pugh score, tumor stage, adverse events after regorafenib treatment, and radiologic assessment for tumor response were extracted. Adverse events were evaluated using National Cancer Institute Common Terminology Criteria for Adverse Events (NCl-CTCAE), version 4.03. Tumor response was evaluated based on radiological assessments such as computed tomography (CT) or magnetic resonance imaging (MRI) using Response Evaluation Criteria In Solid Tumors (RECIST) version 1.1. All laboratory parameters were assessed at the start of regorafenib treatment. Further, inflammatory markers, namely neutrophil to lymphocyte ratio (NLR), platelet to lymphocyte ratio (PLR), systemic immuneinflammation index (SII), were evaluated. The NLR was defined as the neutrophil count divided by the lymphocyte count. The PLR was defined as the platelet counts divided by the lymphocyte counts. The SII 
was calculated as platelet count $\times$ neutrophil count/lymphocyte count [18]. The NLR, PLR and SII scores were stratified into two groups based on each median value.

\section{Statistical analysis}

Categorical variables were analyzed using Pearson's chi-square test. Overall survival (OS) was defined as the time from the start of regorafenib to death. Progression-free survival (PFS) was defined as the time from the start of regorafenib therapy to disease progression. OS and PFS were evaluated using KaplanMeier method and compared using the log-rank test. Univariate and multivariate Cox proportional hazard regression models were used to analyze factors associated with survival. A P value of $<0.05$ was considered statistically significant. All statistical analyses were performed using SPSS statistical software (version 26; SPSS-IBM, Chicago, IL, USA).

\section{Results}

\section{Patient characteristics and regorafenib treatment}

A total of 58 patients who received the regorafenib treatment were included in this study. The baseline characteristics of patients are presented in Table 1. The median age of the patients was 60 years, and most patients ( $n=53,9142 \%$ ) were men. The Child-Pugh scores were 5 and 6 in $65.5 \%$ and $64.5 \%$ of patients, respectively. A total of 15 patients were Barcelona Clinic Liver Cancer (BCLC) stage B and 33 patients as BCLC C. Vascular invasion was noted in 18 (31.0\%) patients and extrahepatic metastasis in $36(62.1 \%)$ patients.

The median duration of regorafenib administration was 2 months (range: 0.5-19 months). All patients experienced at least one treatment-related adverse event. However, no treatment-related deaths was noted. Among the 28 patients who showed progression on regorafenib, 18 (64.3\%) were treated with nivolumab, one with transarterial chemoembolization (TACE), and two with radiotherapy. We evaluated the best tumor response in 51 patients with at least one follow-up radiologic assessment. Six (11.8\%) patients achieved partial response (PR) and $25(49.1 \%)$ patients achieved stable disease (SD). PD was the best response in $20(39.2 \%)$ patients. The disease control rate (DCR) was $60.9 \%$.

\section{Survival Analysis and Predictors of PFS and OS}

The median follow up duration was 5 months. The median OS was 8 months $(95 \%$ confidence interval $[\mathrm{Cl}]=5.761-10.239)$, and the median PFS was 3 months $(95 \% \mathrm{Cl}=0.981-5.019)$. The median times from the start of sorafenib to death was 13 months $(95 \% \mathrm{Cl}=5.4-20.6)$. Table 2 summarizes the results of factors associated with OS. The AFP level (hazard ratio $[\mathrm{HR}]=2.750,95 \% \mathrm{Cl}=1.259-6.010, \mathrm{P}=0.011$ ) and $\mathrm{SII}(\mathrm{HR}=2.211,95 \% \mathrm{Cl}=1.089-4.489, \mathrm{P}=0.028)$ were found to be independent predictors of poor OS in multivariate analysis. Although we also analyzed independent predictors of PFS, no predictive factors were found to be associated with PFS (data not shown).

\section{Prediction of survival using SII}


The OS was significantly lower in patients with high SII $(\geq 340)$ than in patients with low SII $(P=0.013 ;$ Fig. 1A). No difference was noted in PFS between patients with low and high SII ( $P=0.743$; Fig. 1B). We analyzed patient OS using factors that were significant in multivariate analysis, which were SII and AFP level. Categorization of patients based on the SII and AFP level revealed that patients with high SII and AFP level had significantly lower OS than those with low SIl and AFP level ( $P=0.001$; Fig. 2). The correlation values between the SII and clinical features are presented in Table 3 . We found that high SII levels were associated with more advanced BCLC stage $(P=0.036)$.

\section{Discussion}

Recent promising results of new systemic treatments, including tyrosine kinase inhibitors and immunotherapy, have enabled patients with advanced HCC to receive multiple systemic treatments in sequence. Regorafenib, an oral multikinase inhibitor, demonstrated survival benefit over placebo in the phase 3, double-blinded RESORCE study. An exploratory analysis in the RESORCE trial revealed that sorafenib-regorafenib sequential treatment could provide extended survival (> 2 years) to patients with advanced HCC who did not benefit from locoregional theray.[19] After adopting of regorafenib as an initial second-line agent, several real-life reports with good results of regorafenib treatment when progressed on sorafenib were published. Recently, several novel therapeutic agents as second-line therapies after sorafenib failure have shown promising results. Several treatment options are currently available for patients who failed on sorafenib treatment. Therefore, it is important to identify factors to predict treatment response or prognosis.

The present study investigated the predictive factors using systemic inflammatory markers by evaluating the efficacy and safety of sorafenib-regorafenib sequential therapy in patients with advanced HCC. This study revealed that regorafenib was well tolerated and favorable safety in patients with advanced HCC. Regorafenib demonstrated an objective response rate (ORR) of $11.8 \%$, DCR of $60.9 \%$, a median PFS of 3 months, and a median OS of 8 months. These results are similar to those of previous studies [20]. We investigated the prognostic role of systemic inflammatory markers including SII and found that the SII was an independent predictive factor associated with OS. High SII levels at initiation of regorafenib were associated with poor survival.

Previous studies have demonstrated association of inflammation markers with cancer prognosis and elevated SII is associated with poor OS or PFS of patients with cancers. In HCC, many studies have reported relationship between SII and prognosis of patients treated with various treatments [21-23]. As a combined score based on peripheral platelet, lymphocyte and neutrophils counts, the predictive value of SIl for survival may be explained by the role of these immune cells. High SIl usually results from thrombocytosis, neutrophilia and lymphopenia, suggesting a decreased immune response. Inflammation plays essential role in the development of cancer and promotes all stage of tumor progression. Cancer cells are surrounded by stromal cells and immune cells to form tumor microenvironment [24]. Increasing evidences have demonstrated that neutrophilia and thrombocytosis are associated with cancer progression [25-28]. Neutrophils play pro-tumoral roles through multiple mechanisms. Neutrophils can 
enhance cancer cell invasion, proliferation and metastasis by releasing inflammatory mediators such as neutrophil elastase, matrix metalloproteinase-9, and interleukin-8. Neutrophils secrete the proinflammatory factors in the tumor microenvironment, resulting in lymphocyte apoptosis and immunosuppression [29]. Platelets act as multifunctional cells participating in hemostasis, tissue generation and immune response as well as in cancer growth, invasion, and metastasis. Growing evidence had demonstrated that platelets facilitate cancer progression and have a well-defined role in cancer invasion and metastasis [30,31]. Conversely, lymphocytes are known to play a fundamental role in cell-mediated immune response against cancer. Lymphopenia, which reflects the decreased immune surveiilance against cancer, has been reportedly to be associated with poor survival in various solid tumors [32-34]. We hypothesized that cancer therapeutic agents cause immune perturbation and an inflammation-based prognostic factor can reflect a patient's systemic immune status. Thus, due to high platelets and neutrophils levels while low lymphocytes level, a high SII reflected a weak immune response in patients that favor pro-tumoral microenviroment.

The AFP level has been used as a diagnostic criterion and is well known to be correlate with HCC prognosis. High AFP levels are associated with larger tumor, bilobar involvement, vascular invasion, poorly differentiated histology and decreased survival [35]. The AFP level has been included in several HCC prognostic scoring systems [36-38]. High AFP levels are recognised as a poor prognostic factor, and AFP level higher than $400 \mathrm{ng} / \mathrm{mL}$ has been consistently associated with poor prognosis in several HCC treatments $[39,40]$. These results may be elucidated from the relationship between AFP level and VEFGR expression level. VEGF and VEGFR-2-mediated signaling play important roles in angiogenesis and contribute to tumor growth in various cancers, including HCC [41, 42]. Increased AFP levels have been associated with increased VEGFR expression and increased angiogenesis in HCC [43, 44]. In this study, patients with high AFP and SIl showed poor prognosis compared with those with low AFP level and SII. However, no correlation was found between the AFP and SII.

Despite the survival benefit, the overall response of regorafenib in patients with $\mathrm{HCC}$ who progressed on sorafenib treatment are modest and heterogenous because the prognosis of HCC is affected by tumor stage, severity of underlying liver disease and performance status. Moreover, the heterogeneity of the tumor staging itself can lead to a various prognosis. Although tumor staging is an important predictor for prognosis, a more complete understanding of tumor biology and host immune status are required to predict treatment response. This study investigated factors including well-known variable in previous studies, such as tumor staging and tumor markers associated with survival. Our study confirmed that there is still a relevant discrepancy between tumor staging and prognosis, raising the importance of understanding tumor biology and host immune status.

This study has several limitations. First, this study was a retrospective, single-center study with a small number of patients. Second, cutoff value of SII is arbitrary and validation of the cutoff value was not performed. Third, we did not conduct additional experiments to identify the underlying mechanism of the relationship between the SII and survival in patients with advanced HCC received regorafenib treatment. However, despite these limitations, this is the first study according to our knowledge to suggest that SII 
alone can predict the prognosis of patients with advanced HCC who received sorafenib-regorafenib sequential treatment.

\section{Conclusion}

Our data demonstrated that elevated SII is associated with poor OS as well as the possibility of using the AFP level combined with the SII in patients with HCC who received sorafenib-regorafenib sequential treatment. If validated in a larger prospective study, the SII may provide a simple method for identifying patients with poor prognosis. Early identification of this poor prognosis group can provide an opportunity to change treatment strategies to improve patient outcomes.

\section{Abbreviations}

AFP: alpha-Fetoprotein; BCLC: Barcelona Clinic Liver Cancer; HCC: Hepatocellular carcinoma; NLR: neutrophil to lymphocyte ratio; PFS: Progression free survival; PLR: platelet to lymphocyte ratio; OS: Overall survival; PIVKA: Protein induced by vitamin K absence or antagonist-II; SII: Systemic immuneinflammation index

\section{Declarations}

\section{Ethics approval and consent to participate}

The study was approved by the Institutional Review Boards of Pusan National University Yangsan Hospital. Informed consent was waived due to retrospective nature.

\section{Consent for publication}

Not applicable.

\section{Availability of data and materials}

The datasets used and/or analysed during this study are available from the corresponding author on reasonable request.

\section{Competing interests}

The authors have declared no conflicts of interest.

\section{Funding}

Not applicable.

\section{Acknowledgement}

Not applicable. 


\section{Authors' contributions}

$\mathrm{YMH}$ and $\mathrm{MC}$ participated in the conception and design, acquisition of data, analysis and interpretation of data, drafting and writing the manuscript. YMH, KTY and MC participated in analysis and interpretation of data, statistical analysis and critical revision of the manuscript. All authors approved the final manuscript.

\section{References}

1. Global Burden of Disease Liver Cancer C, Akinyemiju T, Abera S, Ahmed M, Alam N, Alemayohu MA, Allen C, Al-Raddadi R, Alvis-Guzman N, Amoako Y et al: The Burden of Primary Liver Cancer and Underlying Etiologies From 1990 to 2015 at the Global, Regional, and National Level: Results From the Global Burden of Disease Study 2015. JAMA Oncol 2017, 3(12):1683-1691.

2. Llovet JM, Ricci S, Mazzaferro V, Hilgard P, Gane E, Blanc JF, de Oliveira AC, Santoro A, Raoul JL, Forner A et al: Sorafenib in advanced hepatocellular carcinoma. N Engl J Med 2008, 359(4):378-390.

3. Bruix J, Qin S, Merle P, Granito A, Huang YH, Bodoky G, Pracht M, Yokosuka O, Rosmorduc O, Breder V et al: Regorafenib for patients with hepatocellular carcinoma who progressed on sorafenib treatment (RESORCE): a randomised, double-blind, placebo-controlled, phase 3 trial. Lancet 2017, 389(10064):56-66.

4. Abou-Alfa GK, Meyer T, Cheng AL, El-Khoueiry AB, Rimassa L, Ryoo BY, Cicin I, Merle P, Chen Y, Park JW et al: Cabozantinib in Patients with Advanced and Progressing Hepatocellular Carcinoma. N Engl J Med 2018, 379(1):54-63.

5. El-Khoueiry AB, Sangro B, Yau T, Crocenzi TS, Kudo M, Hsu C, Kim TY, Choo SP, Trojan J, Welling THR et al: Nivolumab in patients with advanced hepatocellular carcinoma (CheckMate 040): an openlabel, non-comparative, phase 1/2 dose escalation and expansion trial. Lancet 2017, 389(10088):2492-2502.

6. Zhu AX, Finn RS, Edeline J, Cattan S, Ogasawara S, Palmer D, Verslype C, Zagonel V, Fartoux L, Vogel A et al: Pembrolizumab in patients with advanced hepatocellular carcinoma previously treated with sorafenib (KEYNOTE-224): a non-randomised, open-label phase 2 trial. Lancet Oncol 2018, 19(7):940-952.

7. Park JO, Ryoo BY, Yen CJ, Kudo M, Yang L, Abada PB, Cheng R, Orlando M, Zhu AX, Okusaka T: Second-line ramucirumab therapy for advanced hepatocellular carcinoma (REACH): an East Asian and non-East Asian subgroup analysis. Oncotarget 2016, 7(46):75482-75491.

8. Zhu AX, Kang YK, Yen CJ, Finn RS, Galle PR, Llovet JM, Assenat E, Brandi G, Pracht M, Lim HY et al: Ramucirumab after sorafenib in patients with advanced hepatocellular carcinoma and increased alpha-fetoprotein concentrations (REACH-2): a randomised, double-blind, placebo-controlled, phase 3 trial. Lancet Oncol 2019, 20(2):282-296.

9. Wilhelm SM, Carter C, Tang L, Wilkie D, McNabola A, Rong H, Chen C, Zhang X, Vincent P, McHugh M et al: BAY 43-9006 exhibits broad spectrum oral antitumor activity and targets the RAF/MEK/ERK 
pathway and receptor tyrosine kinases involved in tumor progression and angiogenesis. Cancer Res 2004, 64(19):7099-7109.

10. Wilhelm SM, Dumas J, Adnane L, Lynch M, Carter CA, Schutz G, Thierauch KH, Zopf D: Regorafenib (BAY 73-4506): a new oral multikinase inhibitor of angiogenic, stromal and oncogenic receptor tyrosine kinases with potent preclinical antitumor activity. Int J Cancer 2011, 129(1):245-255.

11. Abou-Elkacem L, Arns S, Brix G, Gremse F, Zopf D, Kiessling F, Lederle W: Regorafenib inhibits growth, angiogenesis, and metastasis in a highly aggressive, orthotopic colon cancer model. Mol Cancer Ther 2013, 12(7):1322-1331.

12. Elinav E, Nowarski R, Thaiss CA, Hu B, Jin C, Flavell RA: Inflammation-induced cancer: crosstalk between tumours, immune cells and microorganisms. Nat Rev Cancer 2013, 13(11):759-771.

13. Candido J, Hagemann T: Cancer-related inflammation. J Clin Immunol 2013, 33 Suppl 1:S79-84.

14. Yodying H, Matsuda A, Miyashita M, Matsumoto S, Sakurazawa N, Yamada M, Uchida E: Prognostic Significance of Neutrophil-to-Lymphocyte Ratio and Platelet-to-Lymphocyte Ratio in Oncologic Outcomes of Esophageal Cancer: A Systematic Review and Meta-analysis. Ann Surg Oncol 2016, 23(2):646-654.

15. Lin WF, Zhong MF, Zhang YR, Wang H, Zhao HT, Cheng BB, Ling CQ: Prognostic Role of Platelet-toLymphocyte Ratio in Hepatocellular Carcinoma with Different BCLC Stages: A Systematic Review and Meta-Analysis. Gastroenterol Res Pract 2018, 2018:5670949.

16. Xu Z, Xu W, Cheng H, Shen W, Ying J, Cheng F, Xu W: The Prognostic Role of the PlateletLymphocytes Ratio in Gastric Cancer: A Meta-Analysis. PLoS One 2016, 11(9):e0163719.

17. Ma JY, Ke LC, Liu Q: The pretreatment platelet-to-lymphocyte ratio predicts clinical outcomes in patients with cervical cancer: A meta-analysis. Medicine (Baltimore) 2018, 97(43):e12897.

18. Hu B, Yang XR, Xu Y, Sun YF, Sun C, Guo W, Zhang X, Wang WM, Qiu SJ, Zhou J et al: Systemic immune-inflammation index predicts prognosis of patients after curative resection for hepatocellular carcinoma. Clin Cancer Res 2014, 20(23):6212-6222.

19. Finn RS, Merle P, Granito A, Huang YH, Bodoky G, Pracht M, Yokosuka O, Rosmorduc O, Gerolami R, Caparello $\mathrm{C}$ et al: Outcomes of sequential treatment with sorafenib followed by regorafenib for HCC: Additional analyses from the phase III RESORCE trial. J Hepatol 2018, 69(2):353-358.

20. Facciorusso A, Abd El Aziz MA, Sacco R: Efficacy of Regorafenib in Hepatocellular Carcinoma Patients: A Systematic Review and Meta-Analysis. Cancers (Basel) 2019, 12(1).

21. Yang Z, Zhang J, Lu Y, Xu Q, Tang B, Wang Q, Zhang W, Chen S, Lu L, Chen X: Aspartate aminotransferase-lymphocyte ratio index and systemic immune-inflammation index predict overall survival in HBV-related hepatocellular carcinoma patients after transcatheter arterial chemoembolization. Oncotarget 2015, 6(40):43090-43098.

22. Casadei Gardini A, Scarpi E, Faloppi L, Scartozzi M, Silvestris N, Santini D, de Stefano G, Marisi G, Negri FV, Foschi FG et al: Immune inflammation indicators and implication for immune modulation strategies in advanced hepatocellular carcinoma patients receiving sorafenib. Oncotarget 2016, 7(41):67142-67149. 
23. Wang B, Huang $Y$, Lin $T$ : Prognostic impact of elevated pre-treatment systemic immune-inflammation index (SII) in hepatocellular carcinoma: A meta-analysis. Medicine (Baltimore) 2020, 99(1):e18571.

24. Greten FR, Grivennikov SI: Inflammation and Cancer: Triggers, Mechanisms, and Consequences. Immunity 2019, 51(1):27-41.

25. Ocana A, Nieto-Jimenez C, Pandiella A, Templeton AJ: Neutrophils in cancer: prognostic role and therapeutic strategies. Mol Cancer 2017, 16(1):137.

26. Treffers LW, Hiemstra IH, Kuijpers TW, van den Berg TK, Matlung HL: Neutrophils in cancer. Immunol Rev 2016, 273(1):312-328.

27. Bambace NM, Holmes CE: The platelet contribution to cancer progression. J Thromb Haemost 2011, 9(2):237-249.

28. Gay LJ, Felding-Habermann B: Contribution of platelets to tumour metastasis. Nat Rev Cancer 2011, 11(2):123-134.

29. Wu L, Saxena S, Awaji M, Singh RK: Tumor-Associated Neutrophils in Cancer: Going Pro. Cancers (Basel) 2019, 11(4).

30. Menter DG, Tucker SC, Kopetz S, Sood AK, Crissman JD, Honn KV: Platelets and cancer: a casual or causal relationship: revisited. Cancer Metastasis Rev 2014, 33(1):231-269.

31. Gresele P, Momi S, Malvestiti M, Sebastiano M: Platelet-targeted pharmacologic treatments as anticancer therapy. Cancer Metastasis Rev 2017, 36(2):331-355.

32. Wu ES, Oduyebo T, Cobb LP, Cholakian D, Kong X, Fader AN, Levinson KL, Tanner EJ, 3rd, Stone RL, Piotrowski A et al: Lymphopenia and its association with survival in patients with locally advanced cervical cancer. Gynecol Oncol 2016, 140(1):76-82.

33. Ray-Coquard I, Cropet C, Van Glabbeke M, Sebban C, Le Cesne A, Judson I, Tredan O, Verweij J, Biron P, Labidi I et al: Lymphopenia as a prognostic factor for overall survival in advanced carcinomas, sarcomas, and lymphomas. Cancer Res 2009, 69(13):5383-5391.

34. Cho Y, Park S, Byun HK, Lee CG, Cho J, Hong MH, Kim HR, Cho BC, Kim S, Park J et al: Impact of Treatment-Related Lymphopenia on Immunotherapy for Advanced Non-Small Cell Lung Cancer. Int J Radiat Oncol Biol Phys 2019, 105(5):1065-1073.

35. Gomaa Al, Khan SA, Leen EL, Waked I, Taylor-Robinson SD: Diagnosis of hepatocellular carcinoma. World J Gastroenterol 2009, 15(11):1301-1314.

36. A new prognostic system for hepatocellular carcinoma: a retrospective study of 435 patients: the Cancer of the Liver Italian Program (CLIP) investigators. Hepatology 1998, 28(3):751-755.

37. Borzio M, Dionigi E, Rossini A, Marignani M, Sacco R, De Sio I, Bertolini E, Francica G, Giacomin A, Parisi $\mathrm{G}$ et al: External validation of the ITA.LI.CA prognostic system for patients with hepatocellular carcinoma: A multicenter cohort study. Hepatology 2018, 67(6):2215-2225.

38. Leung TW, Tang AM, Zee B, Lau WY, Lai PB, Leung KL, Lau JT, Yu SC, Johnson PJ: Construction of the Chinese University Prognostic Index for hepatocellular carcinoma and comparison with the TNM 
staging system, the Okuda staging system, and the Cancer of the Liver Italian Program staging system: a study based on 926 patients. Cancer 2002, 94(6):1760-1769.

39. Tangkijvanich P, Anukulkarnkusol N, Suwangool P, Lertmaharit S, Hanvivatvong O, Kullavanijaya P, Poovorawan Y: Clinical characteristics and prognosis of hepatocellular carcinoma: analysis based on serum alpha-fetoprotein levels. J Clin Gastroenterol 2000, 31(4):302-308.

40. Nakazawa T, Hidaka H, Takada J, Okuwaki Y, Tanaka Y, Watanabe M, Shibuya A, Minamino T, Kokubu S, Koizumi W: Early increase in alpha-fetoprotein for predicting unfavorable clinical outcomes in patients with advanced hepatocellular carcinoma treated with sorafenib. Eur $\mathrm{J}$ Gastroenterol Hepatol 2013, 25(6):683-689.

41. Amini A, Masoumi Moghaddam S, Morris DL, Pourgholami MH: The critical role of vascular endothelial growth factor in tumor angiogenesis. Curr Cancer Drug Targets 2012, 12(1):23-43.

42. Tugues S, Koch S, Gualandi L, Li X, Claesson-Welsh L: Vascular endothelial growth factors and receptors: anti-angiogenic therapy in the treatment of cancer. Mol Aspects Med 2011, 32(2):88-111.

43. Yamashita T, Forgues M, Wang W, Kim JW, Ye Q, Jia H, Budhu A, Zanetti KA, Chen Y, Qin LX et al: EpCAM and alpha-fetoprotein expression defines novel prognostic subtypes of hepatocellular carcinoma. Cancer Res 2008, 68(5):1451-1461.

44. Shan YF, Huang YL, Xie YK, Tan YH, Chen BC, Zhou MT, Shi HQ, Yu ZP, Song QT, Zhang QY: Angiogenesis and clinicopathologic characteristics in different hepatocellular carcinoma subtypes defined by EpCAM and alpha-fetoprotein expression status. Med Oncol 2011, 28(4):1012-1016.

\section{Tables}

Table 1. Baseline characteristics of the patients. 


\begin{tabular}{|l|c|}
\hline & $\mathbf{N}=58$ \\
\hline Age (years) & $60(33-86)$ \\
\hline Sex, male & $53(91.4)$ \\
\hline Etiology of HCC & \\
\hline HBV & \\
\hline Others & \\
\hline Child-Pugh score & $38(65.5)$ \\
\hline A5 & $20(34.5)$ \\
\hline A6 & \\
\hline ALBI grade & $26(44.8)$ \\
\hline 1 & $32(55.2)$ \\
\hline 2 & $594(10-9242)$ \\
\hline AFP & $4899(796-25522)$ \\
\hline PIVKA-II & \\
\hline BCLC stage & $15(25.9)$ \\
\hline B & $43(74.1)$ \\
\hline C & $18(31.0)$ \\
\hline Vascular invasion (yes/no) & $36(62.1)$ \\
\hline Extrahepatic meta (yes/no) & $20(34.5)$ \\
\hline Lung & $10(17.2)$ \\
\hline Lymph node & $8(13.8)$ \\
\hline Bone & $2(3.4)$ \\
\hline Adrenal gland & $9(15.5)$ \\
\hline Others & $2.9(1.5-28.1)$ \\
\hline Sorafenib treatment duration (months) & \\
\hline
\end{tabular}

Values are presented as median (interquartile range) or number (\%) of patients. ALBI, albumin-bilirubin; HCC, hepatocellular carcinoma; HBV, hepatitis B virus; BCLC, Barcelona Clinic Liver Cancer; AFP, alpha-fetoprotein; PIVKA, protein induced by vitamin Kabsence or antagonist

Table 2. Univariate and Multivariate COX Regression for Overall Survival

\begin{tabular}{|l|l|c|c|c|}
\hline & \multicolumn{2}{|c|}{ Univariate } & \multicolumn{2}{c|}{ Multivariate } \\
\hline Variables & P value & HR(95\% CI & P value & HR(95\% CI $)$ \\
\hline Age & 0.277 & $0.684(0.345-1.357)$ & & \\
\hline NLR $\geq 2.4$ & 0.362 & $1.375(0.694-2.725)$ & & \\
\hline PLR $\geq 108$ & 0.098 & $1.783(0.898-3.539)$ & & \\
\hline SII $\geq 340$ & 0.020 & $2.308(1.140-4.676)$ & 0.028 & $2.211(1.089-4.489)$ \\
\hline Child Pugh score 6 & 0.248 & $1.549(0.737-3.254)$ & & \\
\hline ALBI grade 2 & 0.055 & $2.004(0.987-4.072)$ & & \\
\hline AFP $\geq 400$ ng/mL & 0.008 & $2.849(1.309-6.200)$ & 0.011 & $2.750(1.259-6.010)$ \\
\hline PIVKA-II $\geq 1000$ mAU/mL & 0.079 & $2.122(0.917-4.913)$ & & \\
\hline BCLC stage C & 0.527 & $0.787(0.375-1.652)$ & & \\
\hline Vascular invasion & 0.171 & $1.652(0.805-3.391)$ & & \\
\hline Extrahepatic metastasis & 0.450 & $0.770(0.390-1.518)$ & & \\
\hline
\end{tabular}

AFP, alpha-fetoprotein; ALBI, albumin-bilirubin; BCLC, Barcelona Clinic Liver Cancer; NLR, neutrophil-to-lymphocyte ratio; PLR, platelet-to-lymphocyte ratio; PIVKA, protein induced by vitamin K absence or antagonist; SII, systemic immune-inflammation index

Table 3. Clinical factors associated with SII 


\begin{tabular}{|c|c|c|c|}
\hline Variables & Low SII $(n=29)$ & High SII $(n=29)$ & P value \\
\hline Age & & & 0.599 \\
\hline$<60$ & $15(51.7)$ & $13(44.8)$ & \\
\hline$\geq 60$ & $14(48.3)$ & $16(55.2)$ & \\
\hline Sex & & & 0.640 \\
\hline Male & $26(89.7)$ & $27(93.1)$ & \\
\hline Female & $3(10.3)$ & $2(6.9)$ & \\
\hline Child Pugh score & & & 0.401 \\
\hline A5 & $18(62.1)$ & $21(72.4)$ & \\
\hline A6 & $11(37.9)$ & $8(27.6)$ & \\
\hline ALBI grade & & & 1.000 \\
\hline 1 & $13(44.8)$ & $13(44.8)$ & \\
\hline 2 & $16(55.2)$ & $16(55.2)$ & \\
\hline $\mathrm{AFP}(\mathrm{ng} / \mathrm{mL})$ & & & 0.597 \\
\hline$<400$ & $14(48.3)$ & $12(41.4)$ & \\
\hline$\geq 400$ & $15(51.7)$ & $17(58.6)$ & \\
\hline PIVKA-II (mAU/mL) & & & 0.240 \\
\hline$<1000$ & $10(34.5)$ & $6(20.7)$ & \\
\hline$\geq 1000$ & $19(65.5)$ & $23(79.3)$ & \\
\hline NLR & & & 0.000 \\
\hline$<2.4$ & $23(79.3)$ & $5(17.2)$ & \\
\hline$\geq 2.4$ & $6(20.7)$ & $24(82.8)$ & \\
\hline PLR & & & 0.000 \\
\hline$<108$ & $12(79.3)$ & $6(20.7)$ & \\
\hline$\geq 108$ & $6(20.7)$ & $23(79.3)$ & \\
\hline BCLC stage & & & 0.036 \\
\hline $\bar{B}$ & $11(37.9)$ & $4(13.8)$ & \\
\hline $\mathrm{C}$ & $18(62.1)$ & $25(86.2)$ & \\
\hline Vascular invasion & & & 1.000 \\
\hline No & $20(69.0)$ & $20(69.0)$ & \\
\hline Yes & $9(31.0)$ & $9(31.0)$ & \\
\hline Extrahepatic metastases & & & 0.104 \\
\hline No & $14(48.3)$ & $8(37.6)$ & \\
\hline Yes & $15(51.7)$ & $21(72.4)$ & \\
\hline
\end{tabular}

Values are presented as number (\%) of patients.

AFP, alpha-fetoprotein; ALBI, albumin-bilirubin; BCLC, Barcelona Clinic Liver Cancer; NLR, neutrophil-to-lymphocyte ratio; PLR, platelet-to-lymphocyte ratio; PIVKA, protein induced by vitamin K absence or antagonist; SII, systemic immune-inflammation index 

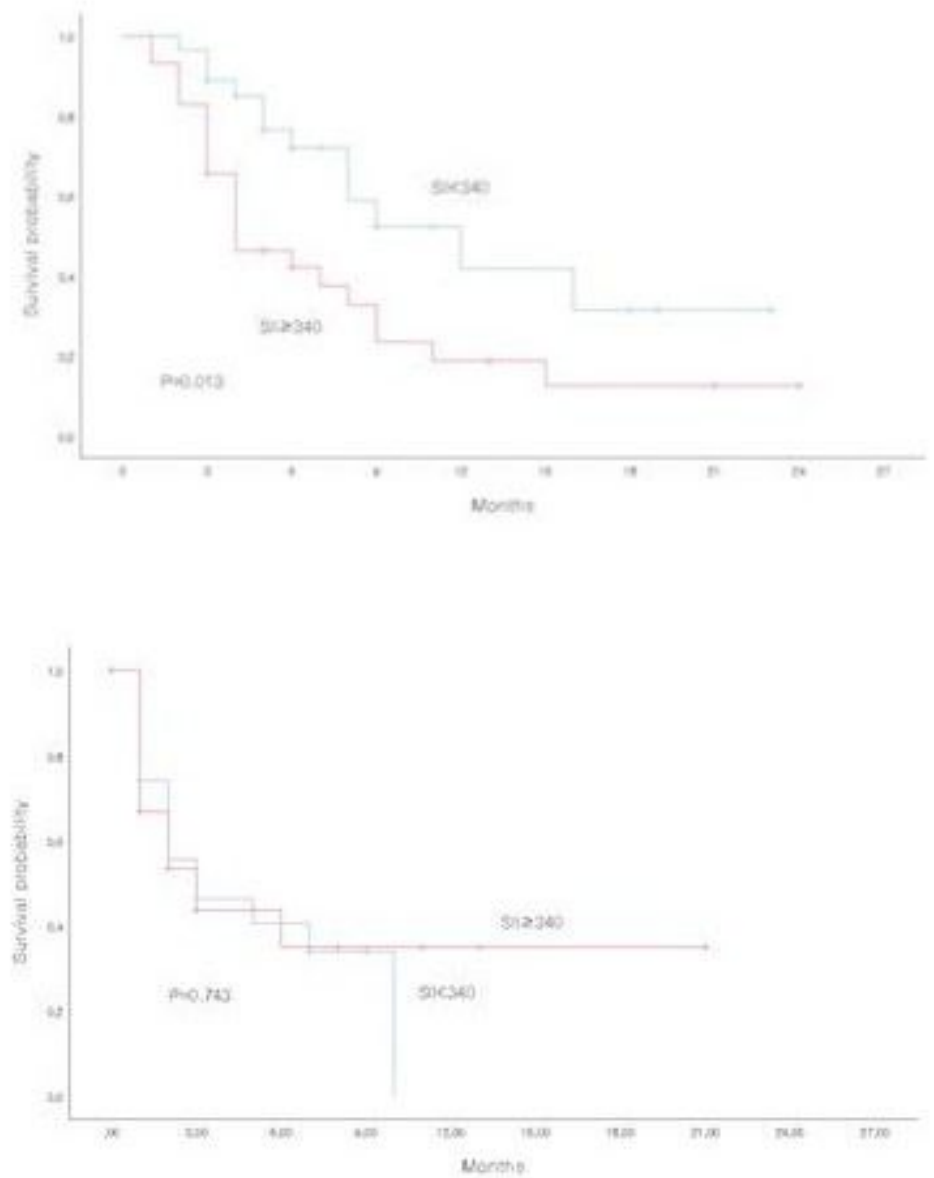

Figure 1

(A - top) Overall survival and (B - bottom) Progression free survival based on systemic immuneinflammation index. 

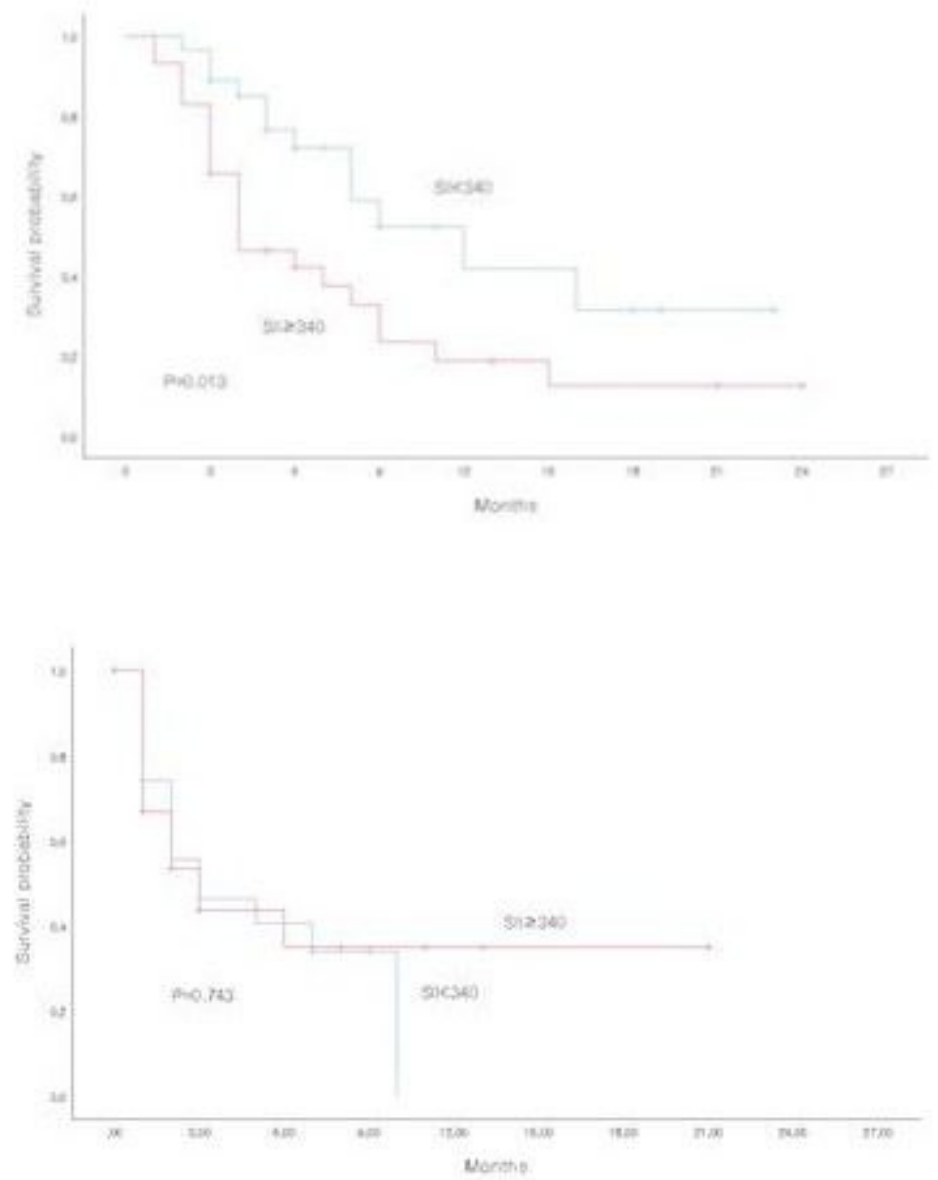

Figure 1

(A - top) Overall survival and (B - bottom) Progression free survival based on systemic immuneinflammation index.

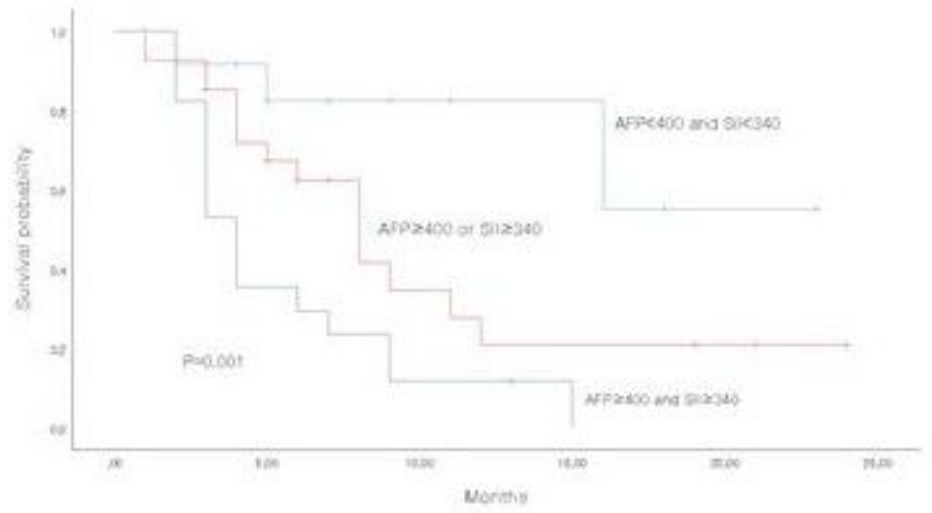

Figure 2

Overall survival based on different levels of systemic immune-inflammation index and alpha-Fetoprotein 


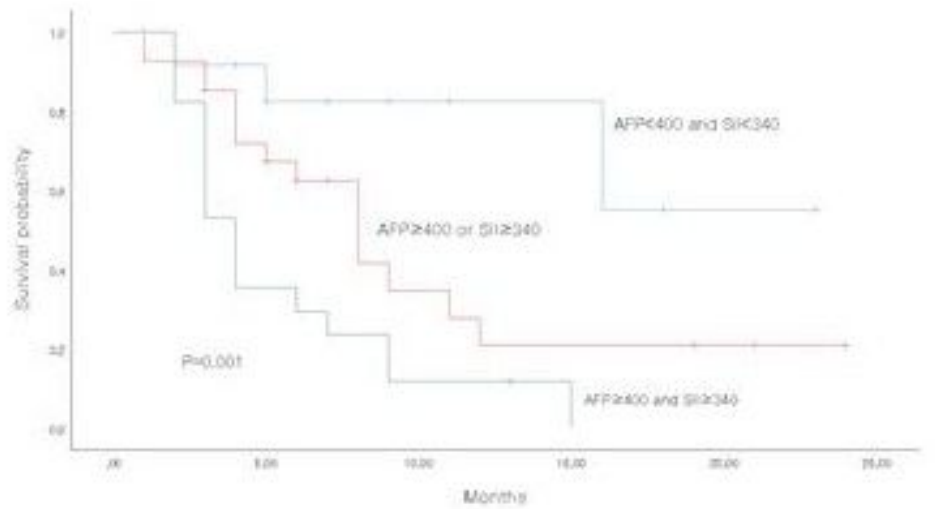

Figure 2

Overall survival based on different levels of systemic immune-inflammation index and alpha-Fetoprotein 